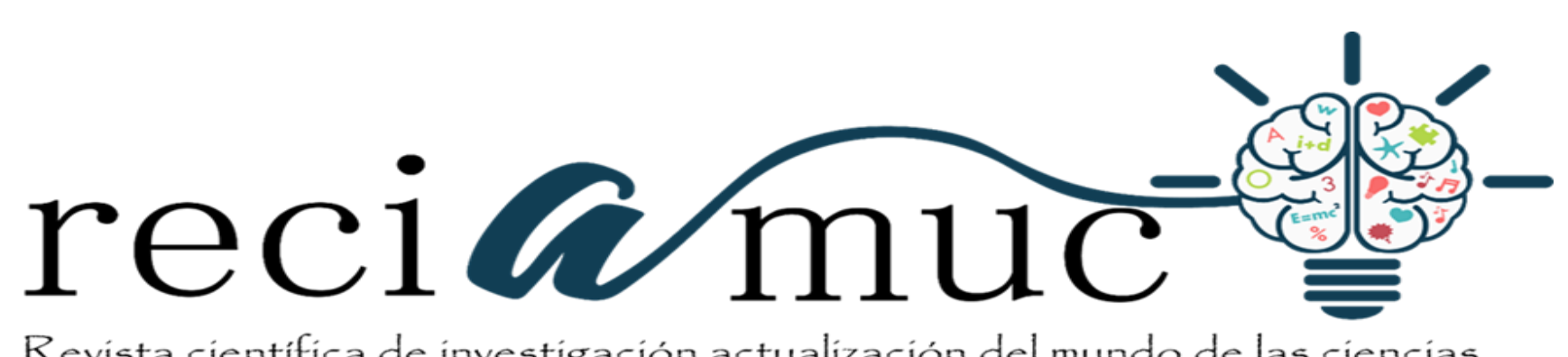

Revista cientifica de investigación actualización del mundo de las ciencias

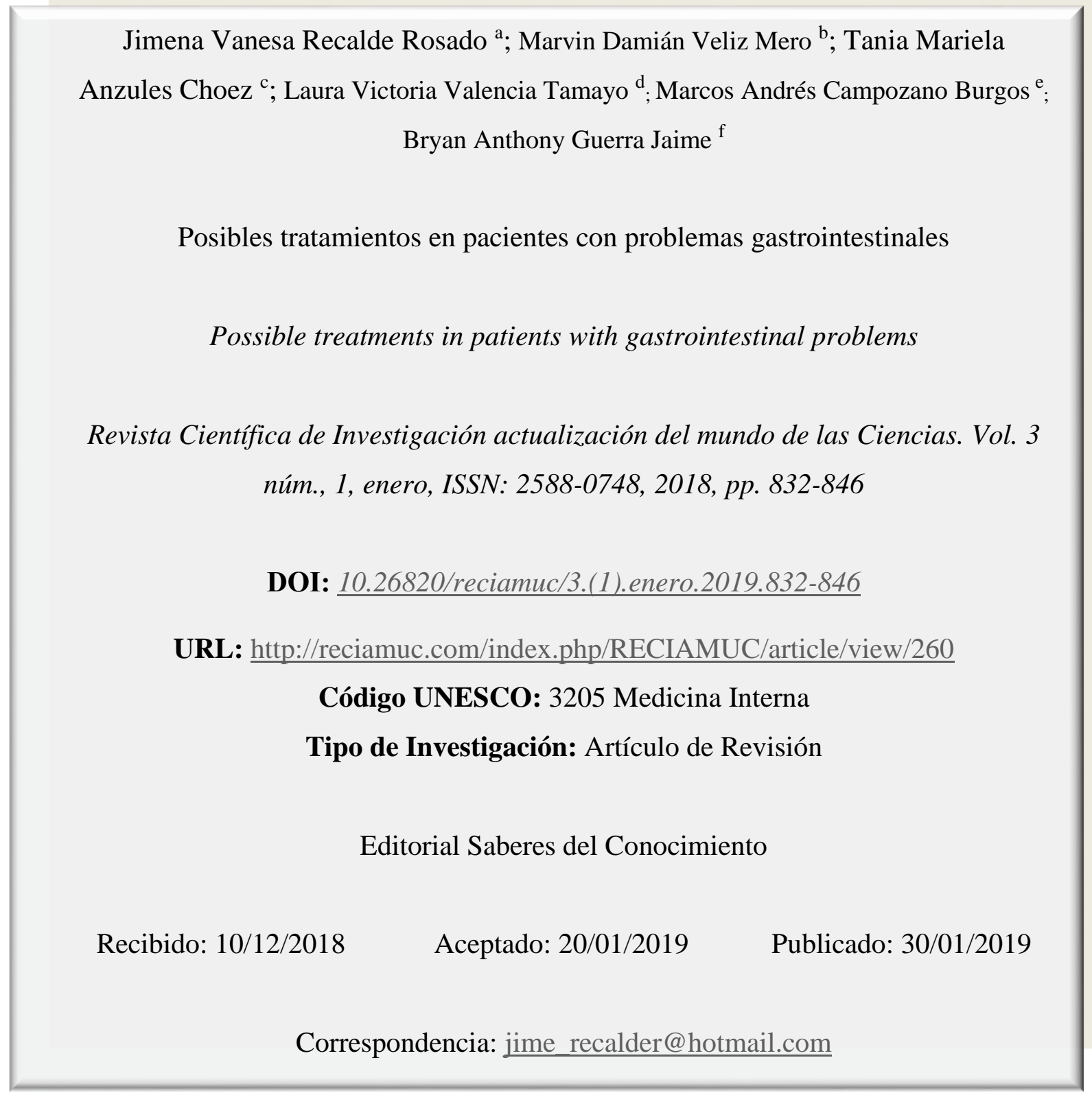
a. Médico Cirujano; jime recalder@hotmail.com; Manta, Ecuador.
b. Médico Cirujano; drmarveliz@ hotmail.com; Manta, Ecuador.
c. Licenciada en Enfermería; tamary 3@ @otmail.com; Portoviejo, Ecuador
d. Médico Cirujano; 1vvt31@ @otmail.com; Manta, Ecuador.
e. Médico Cirujano; andres_bigman25@hotmail.com; Manta, Ecuador.
f. Interno de Medicina Hospital General Manta; bryanjaime@ hotmail.es; Manta, Ecuador. 


\section{Posibles tratamientos en pacientes con problemas gastrointestinales.}

Vol. 3, núm. 1., (2019)

Jimena Vanesa Recalde Rosado; Marvin Damián Veliz Mero; Tania Mariela Anzules Choez; Laura

Victoria Valencia Tamayo; Marcos Andrés Campozano Burgos; Bryan Anthony Guerra Jaime

\section{RESUMEN}

Los trastornos gastrointestinales funcionales (TGFs) siguen siendo un problema frecuente tanto en la edad pediátrica como en la adultez. Estas condiciones fueron reconocidas por primera vez por Apley en 1983 cuando acuñó el término anterior 'dolor abdominal recurrente' (DAR) para un grupo de niños con recurrente o dolor abdominal persistente que interferían con la vida diaria funcional y no tenían etiología orgánica evidente. Los estudios basados en la comunidad de todo el mundo demuestran que el $10 \%$ y el $46 \%$ de todos los niños cumplan las condiciones para DAR. Como TGFs siguen causando cargas emocionales y financieras de las familias afectadas, se están haciendo esfuerzos para categorizar mejor los TGFs como un diagnóstico basado en los síntomas, entender su fisiopatología, e investigar las opciones de tratamiento eficaces. Esta revisión se centra en la dieta, farmacológico, conductual cognitiva, y el tratamiento médico complementario. Debido a la literatura sobre el tratamiento utiliza el término DAR, se usa ese término sabiendo que representa una mezcla de TGFs.

Palabras Claves: Desordenes gastrointestinales, Tratamiento, Síntomas. 


\section{Posibles tratamientos en pacientes con problemas gastrointestinales.}

Vol. 3, núm. 1., (2019)

Jimena Vanesa Recalde Rosado; Marvin Damián Veliz Mero; Tania Mariela Anzules Choez;

Laura Victoria Valencia Tamayo; Marcos Andrés Campozano Burgos; Bryan Anthony Guerra

Jaime

\section{ABSTRACT}

Functional gastrointestinal disorders (TGFs) continue to be a frequent problem in both pediatric and adult age. These conditions were recognized for the first time by Apley in 1983 when he coined the previous term 'recurrent abdominal pain' (ARD) for a group of children with recurrent or persistent abdominal pain that interfered with functional daily life and had no obvious organic etiology. Community-based studies from around the world show that $10 \%$ and $46 \%$ of all children meet the conditions for GIVING. As TGFs continue to cause emotional and financial burdens on affected families, efforts are being made to better categorize TFRs as a diagnosis based on symptoms, understand their pathophysiology, and investigate effective treatment options. This review focuses on diet, pharmacological, cognitive behavioral, and complementary medical treatment. Because the treatment literature uses the term DAR, that term is used knowing that it represents a mixture of TGFs.

Key Words: Gastrointestinal disorders, Treatment, Symptoms. 


\section{Posibles tratamientos en pacientes con problemas gastrointestinales.}

Vol. 3, núm. 1., (2019)

Jimena Vanesa Recalde Rosado; Marvin Damián Veliz Mero; Tania Mariela Anzules Choez; Laura

Victoria Valencia Tamayo; Marcos Andrés Campozano Burgos; Bryan Anthony Guerra Jaime

\section{Introducción.}

En este capítulo, se discuten las enfermedades del tracto gastrointestinal que afectan principalmente áreas distintas a la cavidad oral. Este trabajo no pretende ser una revisión completa de todas las enfermedades que afectan el tracto gastrointestinal; más bien, el énfasis está en los aspectos médicos, el papel del médico como proveedor de atención primaria de salud en la detección de condiciones no diagnosticadas y en el monitoreo del cumplimiento del paciente con la terapia médica recomendada para afecciones gastrointestinales que es probable que se encuentren en la práctica general. Se espera que los trabajadores de la salud reconozcan, diagnostiquen y traten afecciones orales asociadas con enfermedades gastrointestinales, así como brindar atención dental para personas afectadas. Para proporcionar un cuidado dental seguro y apropiado, los dentistas generalmente se preocupan por el diagnóstico correcto de manifestaciones de trastornos gastrointestinales, homeostasis, riesgo de infecciones, acciones e interacciones con medicamentos, la capacidad del paciente para soportar el estrés y el trauma de los procedimientos dentales, y la referencia médica adecuada (cuando sea necesario).

Tanto los dentistas como los gastroenterólogos tienen su principal foco dentro del canal alimentario. La embriogénesis común de la cavidad oral y el tracto gastrointestinal es ocasionalmente reforzado para el médico cuando encuentra quistes de mucosa gástricos heterotópicos en las membranas mucosas orales o en la lengua (Lipsett, Sparnon, \& Byard, 1993) Sin embargo, además de estas anomalías relativamente raras, los caminos de los gastroenterólogos y los dentistas se cruzan con bastante frecuencia en la práctica clínica. El tracto digestivo es un tubo muscular largo que mueve los alimentos y acumula secreciones de la boca hasta el ano, a medida que los alimentos ingeridos se propulsan lentamente a través

de este tracto, el intestino asimila las calorías y nutrientes que se encuentran, esenciales para el establecimiento y mantenimiento de las funciones corporales normales. Proteínas, grasas, carbohidratos, vitaminas, minerales, el agua y los medicamentos ingeridos por vía oral (con y sin receta médica) se digieren en este tracto. Este proceso digestivo depende del hidrólisis de moléculas grandes no absorbibles en pequeñas moléculas a través de enzimas secretadas y la absorción de sustancias a través del revestimiento epitelial del tracto digestivo. Desde allí, las 


\section{Posibles tratamientos en pacientes con problemas gastrointestinales.}

Vol. 3, núm. 1., (2019)

Jimena Vanesa Recalde Rosado; Marvin Damián Veliz Mero; Tania Mariela Anzules Choez;

Laura Victoria Valencia Tamayo; Marcos Andrés Campozano Burgos; Bryan Anthony Guerra

Jaime

sustancias digeridas son transportadas por la sangre a vasos y canales linfáticos a través del cuerpo.

Los contenidos restantes de alimentos no digeridos, típicamente fibra de celulosa, se excreta del tracto digestivo a través del recto y el ano.

La digestión y absorción de los materiales nutrientes depende de:

1. Una concentración óptima de iones de hidrógeno $(\mathrm{pH})$ en el intestino;

2. La presencia de sales biliares conjugadas;

3. Concentraciones adecuadas de enzimas para dividir las grasas, proteínas y carbohidratos;

4. Movilidad intestinal adecuada.

Algunos de los alimentos que ingresan a la sangre desde el tracto digestivo pueden ser utilizados por las células sin ser alterado, sin embargo, la mayoría de los alimentos absorbidos pasa a órganos especiales, donde se transforma en nuevas sustancias que son necesarias para las células. Un tal órgano especial es el hígado, donde se produce este metabolismo intermedio. Además, el tracto gastrointestinal es la vía primaria para la administración de fármacos, la absorción, la biotransformación, la desintoxicación y la excreción. Muchos pacientes dentales requieren farmacoterapia en la que los parámetros farmacocinéticas pueden ser alterados por disfunción gastrointestinal y hepatobiliar. En consecuencia, los proveedores de atención de salud oral deben tener una comprensión global del sistema gastrointestinal y de cómo la función normal y anormal puede afectar la salud de los pacientes.

El sistema digestivo se compone de esófago, estómago, intestino delgado e intestino grueso. Cada uno de estos componentes realiza funciones específicas a medida que las sustancias ingeridas se mueven. A través de las diferentes áreas anatómicas. Adicionalmente, la exocrina, las funciones del páncreas, el hígado y la vesícula biliar se combinan para completar la asimilación de calorías y nutrientes dietéticos.

Este capítulo está organizado de tal manera que se presentan distintos tratamientos para los desórdenes que se pueden encontrar a lo largo del tracto digestivo y se expresa una reseña de los métodos y tratamientos utilizados para la supresión de dichas manifestaciones. 


\section{Posibles tratamientos en pacientes con problemas gastrointestinales.}

Vol. 3, núm. 1., (2019)

Jimena Vanesa Recalde Rosado; Marvin Damián Veliz Mero; Tania Mariela Anzules Choez; Laura

Victoria Valencia Tamayo; Marcos Andrés Campozano Burgos; Bryan Anthony Guerra Jaime

\section{Metodología.}

Para el desarrollo de este proceso investigativo, se plantea como metodología la encaminada hacia una orientación científica particular que se encuentra determinada por la necesidad de indagar en forma precisa y coherente una situación, en tal sentido (Davila, 2015) define la metodología "como aquellos pasos previos que son seleccionados por el investigador para lograr resultados favorables que le ayuden a plantear nuevas ideas".(p.66)

Lo citado por el autor, lleva a entender que el desarrollo de la acción investigativa busca simplemente coordinar acciones enmarcadas en una revisión bibliográfica con el fin de complementar ideas previas relacionadas título del artículo que se está haciendo a través de una revisión de literatura, para así finalmente elaborar un cuerpo de consideraciones generales que ayuden a ampliar el interés propuesto.

\section{Tipo de Investigación.}

Dentro de toda práctica investigativa, se precisan acciones de carácter metodológico mediante las cuales, se logra conocer y proyectar los eventos posibles que la determinan, así como las características que hacen del acto científico un proceso interactivo ajustado a una realidad posible de ser interpretada. En este sentido, se puede decir, que la presente investigación corresponde al tipo documental, definido por Castro (2016), "se ocupa del estudio de problemas planteados a nivel teórico, la información requerida para abordarlos se encuentra básicamente en materiales impresos, audiovisuales y /o electrónicos”. (p.41).

En consideración a esta definición, la orientación metodológica permitió la oportunidad de cumplir con una serie de actividades inherentes a la revisión y lectura de diversos documentos donde se encontraron ideas explicitas relacionadas con los tópicos encargados de identificar a cada característica insertada en el estudio. Por lo tanto, se realizaron continuas interpretaciones con el claro propósito de revisar aquellas apreciaciones o investigaciones propuestas por diferentes investigadores relacionadas con el tema de interés, para luego dar la respectiva argumentación a los planteamientos, en función a las necesidades encontradas en la indagación. 


\section{Posibles tratamientos en pacientes con problemas gastrointestinales.}

Vol. 3, núm. 1., (2019)

Jimena Vanesa Recalde Rosado; Marvin Damián Veliz Mero; Tania Mariela Anzules Choez;

Laura Victoria Valencia Tamayo; Marcos Andrés Campozano Burgos; Bryan Anthony Guerra Jaime

\section{Fuentes Documentales.}

El análisis correspondiente a las características que predomina en el tema seleccionado, llevan a incluir diferentes fuentes documentales encargadas de darle el respectivo apoyo y en ese sentido cumplir con la valoración de los hechos a fin de generar nuevos criterios que sirven de referencia a otros procesos investigativos. Para (CASTRO, 2016) las fuentes documentales incorporadas en la investigación documental o bibliográfica, "representa la suma de materiales sistemáticos que son revisados en forma rigurosa y profunda para llegar a un análisis del fenómeno".(p.41). Por lo tanto, se procedió a cumplir con la realización de una lectura previa determinada para encontrar aquellos aspectos estrechamente vinculados con el tema, con el fin de explicar mediante un desarrollo las respectivas apreciaciones generales de importancia.

\section{Técnicas para la Recolección de la Información.}

La conducción de la investigación para ser realizada en función a las particularidades que determinan a los estudios documentales, tiene como fin el desarrollo de un conjunto de acciones encargadas de llevar a la selección de técnicas estrechamente vinculadas con las características del estudio. En tal sentido, (Bolívar, 2015), refiere, que es "una técnica particular para aportar ayuda a los procedimientos de selección de las ideas primarias y secundarias”. (p. 71).

Por ello, se procedió a la utilización del subrayado, resúmenes, fichaje, como parte básica para la revisión y selección de los documentos que presentan el contenido teórico. Es decir, que mediante la aplicación de estas técnicas se pudo llegar a recoger informaciones en cuanto a la revisión bibliográfica de los diversos elementos encargados de orientar el proceso de investigación. Tal como lo expresa, (Bolívar, 2015) "las técnicas documentales proporcionan las herramientas esenciales y determinantes para responder a los objetivos formulados y llegar a resultados efectivos" (p. 58). Es decir, para responder con eficiencia a las necesidades investigativas, se introdujeron como técnica de recolección el método inductivo, que hizo posible llevar a cabo una valoración de los hechos de forma particular para llegar a la explicación desde una visión general.

Asimismo, se emplearon las técnicas de análisis de información para la realización de la investigación que fue ejecutada bajo la dinámica de aplicar diversos elementos encargados de 


\section{Posibles tratamientos en pacientes con problemas gastrointestinales.}

Vol. 3, núm. 1., (2019)

Jimena Vanesa Recalde Rosado; Marvin Damián Veliz Mero; Tania Mariela Anzules Choez; Laura

Victoria Valencia Tamayo; Marcos Andrés Campozano Burgos; Bryan Anthony Guerra Jaime

determinar el camino a recorrer por el estudio, según, (Bolívar, 2015) las técnicas de procesamiento de datos en los estudios documentales "son las encargadas de ofrecer al investigador la visión pasos que debe cumplir durante su ejercicio, cada una de ellas debe estar en correspondencia con el nivel a emplear" (p. 123). Esto indica, que para llevar a cabo el procesamiento de los datos obtenidos una vez aplicado las técnicas seleccionadas, tales como: fichas de resumen, textual, registros descriptivos entre otros, los mismos se deben ajustar al nivel que ha sido seleccionado.

\section{Resultados}

\section{Intervención dietética}

\section{Las dietas bajas en lactosa}

En un estudio les dieron a 21 niños una dieta libre de lactosa durante 2 semanas. Durante el siguiente período de 2 semanas o bien solución de lactosa o placebo se administraron en un ensayo cruzado doble ciego. No se observaron diferencias en los síntomas de dolor en ninguno de los grupos (Huertas-Ceballos, Macarthur, \& Logan, 2002). En otro estudio encontraron una tolerancia a la lactosa anormal de la prueba oral $(2 \mathrm{~g} / \mathrm{kg}$ ) en 21 de 69 niños con DAR (síntomas de calambres, dolor, hinchazón, gases, diarrea o deposiciones sueltas) documentados por un aumento despuntada de la glucosa en sangre de $<20 \mathrm{mg} / \mathrm{ml}$ (Lebenthal, Rossi, Nord, \& Branski, 1981). En tres sucesivos ensayos cegados de 6 semanas (fórmula de leche de vaca que contiene lactosa, a base de proteína de soja fórmula no la lactosa, y de la dieta normal y el consumo de leche), la frecuencia del dolor se incrementó en 10/21 después de la fórmula lactosa y en 7/21 en la soja sin lactosa. En los niños que se sometieron a ensayo de pequeña biopsia de la mucosa y de la lactasa intestinal, la prevalencia de la insuficiencia de lactasa fue similar en DAR y de control de los niños (8/26 en comparación con 16/61). A pesar de sus limitaciones, estos dos estudios sugieren que la intolerancia a la lactosa puede desempeñar un papel en la producción de síntomas en algunos, pero no la mayoría de los niños con DAR. 


\section{Posibles tratamientos en pacientes con problemas gastrointestinales.}

Vol. 3, núm. 1., (2019)

Jimena Vanesa Recalde Rosado; Marvin Damián Veliz Mero; Tania Mariela Anzules Choez;

Laura Victoria Valencia Tamayo; Marcos Andrés Campozano Burgos; Bryan Anthony Guerra Jaime

Fibra dietética

En un estudio con 40 niños entre 3 a 15 años aleatorizados para recibir una galleta con un contenido de fibra de psyllium de 2\% (placebo) o 66\% (tratamiento) y se observaron diferencias entre los grupos en el número medio de episodios de dolor (Huertas-Ceballos, Macarthur, \& Logan, 2002). Este mismo autor con niños asignados al azar para comer galletas con o sin fibra de maíz y tomó nota de 13 de 26 niños en el grupo de fibra suplementado tenían una reducción del $50 \%$ en la frecuencia de los ataques $(P=0,04)$. Sin embargo, utilizando un análisis odds ratio, en la revisión se encontraron diferencias significativas entre los grupos.

Todos los grupos mejoraron sus síntomas, aunque no se sabe si la respuesta en el grupo de fibras por sí sola podría haber resultado de un efecto placebo. (Paulo, Amancio, de Morais, \& Tabacow, 2006) evaluaron macronutrientes en la dieta y la ingesta de fibra en una evaluación retrospectiva de la sección transversal de DAR. Los pacientes se dividieron en tres grupos: 1) DAR solamente; 2) DAR con estreñimiento durante 3 meses o más; y 3) los niños sin DAR que sirvieron como controles. Aunque no hubo diferencias significativas en la ingesta de macronutrientes, la ingesta de fibra fue mayor en el grupo de control en comparación con los dos grupos de DAR. En el grupo de control en comparación con los dos grupos de DAR, 51\% en comparación con $78 \%$, respectivamente, informaron una ingesta menor que el valor mínimo recomendado $(\mathrm{P}=0,021)$. Este estudio sugiere que una dieta baja en fibra actúa como un factor de riesgo para el DAR.

La fibra puede tener efectos beneficiosos sobre la motilidad del tracto gastrointestinal y la sensibilidad, además de servir como un ablandamiento de heces y agente de carga para aliviar algunos de los factores que contribuyen a la hipótesis de DAR incluyendo estreñimiento. Aunque los estudios han demostrado claros beneficios de la fibra suplementaria en adultos, se necesitan más estudios para delinear su papel beneficioso, en su caso, en el tratamiento de DAR en los niños. Es poco probable que la fibra soluble haga exacerbar los síntomas, y puede proporcionar alivio de los síntomas en algunos niños. Un ensayo de $0,75 \mathrm{~g}$ por año de edad por día toma en cuenta que los niños en este grupo de edad ( 7 a 18 años) reciben aproximadamente $14 \mathrm{a} 16 \mathrm{~g} / \mathrm{d}$ de fibra en la dieta. 


\section{Posibles tratamientos en pacientes con problemas gastrointestinales.}

Vol. 3, núm. 1., (2019)

Jimena Vanesa Recalde Rosado; Marvin Damián Veliz Mero; Tania Mariela Anzules Choez; Laura

Victoria Valencia Tamayo; Marcos Andrés Campozano Burgos; Bryan Anthony Guerra Jaime

\section{Dieta baja en fructosa.}

La fructosa, especialmente en forma de jarabe de maíz alto en fructosa, se ha pensado para causar dolor abdominal crónico. La fructosa se consume de forma ubicua por los niños como un edulcorante común utilizado en refrescos, zumos, dulces, e incluso frutas. La fructosa, como lactosa, cuando es mal absorbida por los pequeño lumen intestinales, puede causar una diarrea osmótica y también sirven como un sustrato para la fermentación bacteriana del colon, y por lo tanto, la producción de gas y posterior dolor abdominal. (Gomara, Halata, \& Newman, 2008) encontraron 11 de 32 niños con TGFs por fructosa mal absorbida. Síntomas gastrointestinales tales como náuseas, hinchazón, y dolor abdominal también fueron más frecuentes en el grupo de dosis más alta fructosa $(\mathrm{P}<0,05)$. Aunque el diseño del estudio tenía limitaciones, un ensayo de una baja o ninguna dieta fructosa durante 2 semanas pueden resultar beneficiosos en algunos niños.

\section{Tratamiento farmacológico}

\section{Los antidepresivos}

Aunque no del todo comprendidas, el mecanismo para TGFs ha sugerido involucrar a la desregulación del sistema nervioso entérico y central que afecta la sensación intestinal y la motilidad. Los medicamentos utilizados para tratar la depresión, la ansiedad y las convulsiones se han convertido en agentes cada vez más atractivos para su uso en TGFs porque actúan sobre el sistema nervioso central y periférico para modular el estado de ánimo, visceral, y el dolor neuropático, así como la función autonómica, en parte, a través de efectos anticolinérgicos.

Antidepresivos tales como antidepresivos tricíclicos (ATC), inhibidores de la recaptación de serotonina (ISRS), e inhibidores de la captación de monoamina mejoran los síntomas de IBS en los adultos (Lebel, 2008). Los antidepresivos actúan disminuyendo la percepción del dolor, mejorar el estado de ánimo y trastornos del sueño, y tal vez la regulación de la motilidad. ATCs (a través de un efecto anticolinérgico) y los ISRS mejoran la diarrea al desacelerar el tránsito y el estreñimiento por acelerar el tiempo de tránsito, respectivamente. Los metanálisis la 


\section{Posibles tratamientos en pacientes con problemas gastrointestinales.}

Vol. 3, núm. 1., (2019)

Jimena Vanesa Recalde Rosado; Marvin Damián Veliz Mero; Tania Mariela Anzules Choez;

Laura Victoria Valencia Tamayo; Marcos Andrés Campozano Burgos; Bryan Anthony Guerra Jaime

evaluación de los efectos de los antidepresivos en adultos con TGFs han demostrado un efecto analgésico beneficioso independiente de mejoras en el estado de ánimo.

Los ATC han sido los agentes psicotrópicos más estudiados para TGFs en adultos, incluyendo el SII y FD. ATCs en comparación a los ISRS han demostrado la reducción del dolor más pronunciado en el dolor neuropático crónico en modelos animales y se cree que son más eficaces en los sistemas nerviosos centrales que en los periféricos. Sus efectos secundarios, más a menudo en los ATC terciaria (amitriptilina, imipramina) que en las aminas secundarias (desipramina, nortriptilina), incluyen sedación, efectos anticolinérgicos (estreñimiento y retención urinaria), y efectos sobre el sistema nervioso central (insomnio y agitación). En un reciente ensayo controlado con placebo amitriptilina se evaluó en 33 adolescentes con TGFs, en comparación con el placebo, los sujetos tratados con amitriptilina habían mejorado la calidad de vida de las puntuaciones, menos cuadrante inferior derecho y el dolor periumbilical, y la diarrea relacionada con el TGF (Bahar, Collins, Steinmetz, \& Ament, 2008) Sin embargo, estos resultados deben ser replicados en un estudio más amplio.

ISRS bloquean la recaptación de 5-hidroxitriptamina (5-HT), el aumento de su concentración en las terminaciones nerviosas presinápticas. Aunque los ISRS se han demostrado reducir el dolor neuropático asociado con el dolor de espalda y las migrañas, el efecto sobre el dolor relacionado a trastornos gastrointestinales en adultos es menos clara. El beneficio de los ISRS ha demostrado estar relacionada con la mejora de la sensación general de bienestar, la reducción de la ansiedad derivada de los síntomas relacionados con problemas gastrointestinales, el tratamiento de comorbilidades psiquiátricas, y aumentar las propiedades analgésicas de los ATC cuando se utiliza en combinación. Sin embargo, la propensión ISRS hacia causando diarrea hace que sea una terapia potencial para TGF relacionados con estreñimiento.

Hay una escasez de estudios en pacientes pediátricos con TGFs que evalúan el tratamiento farmacológico. Debido a que los estudios disponibles son pocos y no han sido replicados, la verdadera eficacia de estos fármacos para en niños aún no se ha dilucidado. Sin embargo, en ausencia de datos definitivos, gastroenterólogos pediátricos utilizan algunos de estos 


\section{Posibles tratamientos en pacientes con problemas gastrointestinales.}

Vol. 3, núm. 1., (2019)

Jimena Vanesa Recalde Rosado; Marvin Damián Veliz Mero; Tania Mariela Anzules Choez; Laura

Victoria Valencia Tamayo; Marcos Andrés Campozano Burgos; Bryan Anthony Guerra Jaime

medicamentos empíricamente cuando los niños cumplen los criterios diagnósticos de TGFs, cada vez más se da lugar a extensas pruebas de laboratorio, radiografías y pruebas endoscópica. Por ejemplo, un ensayo de un inhibidor de la bomba antagonista del receptor $\mathrm{H} 2 \mathrm{o}$ de protones se puede utilizar sobre una base empírica durante al menos 2 semanas, con poco riesgo. En los niños con retraso del vaciamiento gástrico presunta, una prueba de 1 semana de la eritromicina puede ser útil (1 a $3 \mathrm{mg} / \mathrm{kg}$ de tres a cuatro veces por día).

\section{Las intervenciones psicológicas.}

Aunque la etiología de TGFs no se ha aclarado por completo, es ampliamente aceptado que esta entidad es multifactorial con el estrés externo y los factores ambientales juegan un cierto papel. Debido a que el tratamiento con dieta o farmacológicos agentes solos ha sido parcialmente exitosas intervenciones psicológicas, como la terapia cognitivo conductual (TCC), la intervención familiar, la distracción, la hipnoterapia y biorretroalimentación se han instituido con eficacia prometedora. (Bursch, 2008)

La terapia familiar incluye la formación de la familia y los padres para apoyar al paciente en funcionamiento de forma independiente a través de auto-afrontamiento y habilidades de autogestión, para desarrollar una mayor tolerancia de los síntomas y reducir la discapacidad y la dependencia que permite en el niño. Psicoterapia para el niño, incluyendo la terapia cognitiva conductual, permite que el niño aprenda técnicas de resolución de problemas y un mejor afrontamiento, identificar los factores desencadenantes, y eliminar o mejorar las reacciones de mala adaptación a los mismos. Las técnicas de relajación y de distracción han demostrado disminuir la percepción del dolor, lo que aumenta la tolerancia al dolor.

Hipnoterapia, un tratamiento efectivo para el TGFs en los adultos, ha sido recientemente estudiado en niños de 8 a 18 años. Aunque las puntuaciones de intensidad del dolor y de frecuencia disminuyeron significativamente en ambos grupos en comparación con el valor basal, en el grupo de hipnoterapia ambas puntuaciones disminuyeron significativamente en comparación con la terapia estándar médico ( $\mathrm{P}<0,002$ para la intensidad del dolor y $\mathrm{P}<0,001$ para la frecuencia del dolor). Las intervenciones psicológicas forman un pilar de la terapia para 


\section{Posibles tratamientos en pacientes con problemas gastrointestinales.}

Vol. 3, núm. 1., (2019)

Jimena Vanesa Recalde Rosado; Marvin Damián Veliz Mero; Tania Mariela Anzules Choez;

Laura Victoria Valencia Tamayo; Marcos Andrés Campozano Burgos; Bryan Anthony Guerra Jaime

niños con TGFs. Es importante reconocer el objetivo principal es ayudar a los niños a lidiar con su dolor. Se recomiendan estas intervenciones por sus efectos fisiológicos no en la creencia de que el dolor se deriva de problemas psicológicos o psicosomáticos primarios.

\section{Medicina Complementaria y Alternativa.}

Según lo definido por el Centro Nacional de medicina complementaria y alternativa, complementaria y medicina alternativa "es un grupo de diversos sistemas médicos y de cuidado de la salud, prácticas y productos que no se considera actualmente que es parte de la medicina convencional." La medicina convencional se define como que se practica por personas que poseen títulos médicos, los títulos de doctores en osteopatía y otros trabajadores de la salud aliados. La medicina complementaria se utiliza junto con la medicina convencional mientras que la medicina alternativa se utiliza en lugar de la medicina convencional.

Aproximadamente $36 \%$ a $41 \%$ de los niños con quejas GI utilizar medicina complementaria cada año, incluyendo los siguientes métodos comúnmente buscadas: medicinas a base de hierbas, la terapia de masaje, y la acupuntura.25 Hay pocos datos para apoyar el uso de la terapia de masaje o la acupuntura.

\section{Los probióticos}

Los probióticos, definidos como microorganismos vivos capaces de sobrevivir y la inducción de un efecto beneficioso en el huésped, son otra forma de terapia con eficacia potencial en el tratamiento de TGFs. Se cree que la flora entérica normales, por sus atributos inmunológicas y metabólicas, contribuyen al homeostasis del tracto GI y regular la inflamación de la mucosa y la inmunidad. IBS se ha documentado que ocurrir en individuos después de que cualquiera infección GI o la terapia con antibióticos, los cuales interrumpen normal de homeostasis flora entérica. Se cree que los probióticos a actuar compitiendo metabólicamente con patógenos, la mejora de la barrera mucosa del intestino, y / o alterar la respuesta inflamatoria intestinal. En este punto, la eficacia de los probióticos en TGFs sigue siendo incierto. Ensayos de alta calidad, faltan en pediatría y pocas conclusiones se pueden extraer como diferentes métodos, 


\section{Posibles tratamientos en pacientes con problemas gastrointestinales.}

Vol. 3, núm. 1., (2019)

Jimena Vanesa Recalde Rosado; Marvin Damián Veliz Mero; Tania Mariela Anzules Choez; Laura

Victoria Valencia Tamayo; Marcos Andrés Campozano Burgos; Bryan Anthony Guerra Jaime

formulaciones de probióticos, dosis y diferentes medidas de resultado se han utilizado hasta el momento.

\section{Conclusión.}

A pesar de una búsqueda continua por un efecto farmacológico "bala mágica” para TGFs, cada vez es más claro que sólo una mejor comprensión de la interacción de mecanismos biopsicosociales (componentes fisiológicos, psicológicos y de comportamiento) que contribuyen al desarrollo de TGFs dará lugar a tratamientos más eficaces. A medida que cada entidad de la enfermedad se vuelve define mejor, reconocemos la contribución de factores tales como la motilidad mal regulada, hipersensibilidad visceral, la infección, la inflamación, la psicología y el comportamiento en la patogénesis de estos trastornos. Es probable que, en el futuro, como ahora, el tratamiento eficaz debe ser personalizado para adaptarse proceso de la enfermedad de cada paciente y la sintomatología específica.

\section{Bibliografía.}

Bahar, R., Collins, B., Steinmetz, B., \& Ament, M. (2008). Double-blind placebo-controlled trial of amitriptyline for the treatment of irritable bowel syndrome in adolescents. J Pediatr, 685-689.

Bolívar, J. (2015). Investigación Documental. México. Pax.

Bursch, B. (2008). Psychological/cognitive behavioral treatment of childhood functional abdominal pain and irritable bowel syndrome. J Pediatr Gastroenterol Nutr, 706-707.

Castro, J. (2016). Técnicas Documentales. México. Limusa.

Davila, A. (2015). Concepto de terminos cientificos. Caracas: Oasis.

Gomara, R., Halata, M., \& Newman, L. (2008). Fructose intolerance in children presenting with abdominal pain. J Pediatr Gastroenterol Nutr, 303-308. 


\section{Posibles tratamientos en pacientes con problemas gastrointestinales.}

Vol. 3, núm. 1., (2019)

Jimena Vanesa Recalde Rosado; Marvin Damián Veliz Mero; Tania Mariela Anzules Choez;

Laura Victoria Valencia Tamayo; Marcos Andrés Campozano Burgos; Bryan Anthony Guerra

Jaime

Huertas-Ceballos, A., Macarthur, C., \& Logan, S. (2002). Dietary interventions for recurrent abdominal pain (RAP) in childhood. Cochrane Database Syst Rev .

Lebel, A. (2008). Pharmacology. J Pediatr Gastroenterol Nutr, 703-705.

Lebenthal, E., Rossi, T., Nord, K., \& Branski, D. (1981). Recurrent abdominal pain and lactose absorption in children. Pediatrics, 828-832.

Lipsett, J., Sparnon, A., \& Byard, R. (1993). Embryogenesis of enterocystomas-enteric duplication cysts of the tongue. Oral Surg Oral Med Oral Pathol, 626-630.

Paulo, A., Amancio, O., de Morais, M., \& Tabacow, K. (2006). Low-dietary fiber intake as a risk factor for recurrent abdominal pain in children. Eur J Clin Nutr, 823-827. 